\title{
Desarrollo de un modelo de bajo costo de punción tiroidea y pauta de evaluación para su entrenamiento
}

\author{
Development of a low-cost thyroid puncture model \\ and evaluation guideline for training \\ Javier Vela U, ${ }^{*}$ Cristián Jarry T, ${ }^{*}$ Caterina Contreras B, ${ }^{*}$ Lorena Mosso G, ${ }^{\ddagger}$ \\ Julián Varas C,* Nicole Lustig $\mathrm{F}^{\ddagger}$
}

Palabras clave:

Punción tiroidea, ecografía, simulación.

Keywords: Thyroid punction, simulation, ecography.

\section{Declaración de}

Conflicto de intereses: Los autores declaran no tener ningún conflicto de intereses.

* Centro de Simulación y Cirugía Experimental.

¥ Departamento de

Endocrinología.

Pontificia Universidad Católica de Chile.

Recibido: $12 / 07 / 2019$ Aceptado: 30/07/2019

\section{RESUMEN}

Introducción: Los nódulos tiroideos son un problema clínico frecuente en cuya solución, la punción mediante una aguja fina guiada por ecografía, juega un rol fundamental. El entrenamiento en esta técnica aumenta su tasa diagnóstica. Objetivos: Desarrollar un modelo de punción tiroidea de bajo costo y una pauta de cotejo para su evaluación. Material y métodos: Se consultó la literatura médica existente y a los expertos locales en punción tiroidea. Se elaboró un modelo tiroideo tras la iteración por expertos, los cuales evaluaron mediante un cuestionario en línea los aspectos como ecogenicidad, puncionabilidad, consistencia, similitud anatómica y costos del modelo. La pauta de evaluación se construyó con base en el consenso de expertos determinando los ítems fundamentales para el procedimiento. Resultados: Se logró construir un modelo con aceptable puncionabilidad, ecogenicidad, consistencia y similitud anatómica según los expertos. El costo de cada modelo es alrededor de 2,6 dólares, tiene una durabilidad aproximada de cuatro semanas y límite de punciones, según la cantidad de nódulos. Se elaboró una pauta de 12 ítems que evalúa aspectos técnicos y clínicos. Esta pauta fue creada para ser aplicada tanto en un ambiente simulado como en el paciente real. Conclusión: En este trabajo se presenta un modelo de punción tiroidea de bajo costo y de fácil replicabilidad junto a una pauta para evaluar el entrenamiento, con miras a desarrollar en el futuro un programa de entrenamiento validado para punción tiroidea.

\section{ABSTRACT}

Introduction: Thyroid nodules are a frequent clinical problem, for which ecography-guided fine needle puncture is an essential tool. Studies show that investing hours on practicing this technique improves the diagnostic outcomes. Objectives: To develop a low-cost thyroid puncture simulation model, and its assessment tool. Material and methods: Both existing literature and experts experience were consulted. An iteration process was conducted for the elaboration of the thyroid puncture model, with constant expert consultation. The following parameters were assessed: echogenicity, punctionability, consistency, anatomic resemblance, and cost. The assessment tool was built from expert consensus with the election of key items for the procedure. Results: A model achieving an acceptable level of echogenicity, punctionability, consistency and anatomic resemblance was built, and approved by local experts. The cost per model was USD 2,6, with an average duration of four weeks. Punction attempts depend on the number of nodules added to each model. The assessment tool developed consisted on twelve items related to technical and clinical aspects of the procedure. This rating scale was created for both clinical and simulated environments. Conclusion: We present a thyroid puncture model, which is both lowcost and easily reproduced, along with its assessment tool. Future challenges consist on developing a validated training program based on this model.

\section{INTRODUCCIÓN}

L os nódulos tiroideos son un problema clínico frecuente, se estima una prevalencia de nódulos tiroideos palpables de 4 a $7 \%$ en población general, ${ }^{1}$ cifra que aumenta de 19 a $67 \%$ con el uso masivo de ecografía, se presenta con mayor frecuencia en los adultos mayores, llegando de 50 a $70 \%$ en personas mayores de 60 años. ${ }^{1-3}$ La gran mayoría de los nódulos tiroideos son benignos y no crecen de forma significativa con el tiempo, por lo que no es recomendable hacer pesquisa de nódulos tiroideos en la población general. Sin embargo, en cierto grupo existe la necesidad de descartar el cáncer de tiroides, lo cual ocurre en 5 a 10\% de 
los nódulos, ${ }^{4,5}$ dependiendo de factores como la edad, género, exposición a la radiación en la infancia e historia familiar, entre otros.

En el enfrentamiento clínico de esta patología, la biopsia mediante la Punción y Aspiración con Aguja Fina (PAAF) se ha convertido en el estándar para el diagnóstico de los nódulos de tamaño superior a 1-1.5 cm, según sus características ecográficas. ${ }^{6}$ Pese a su alta prevalencia, el proceso diagnóstico de esta condición suele conllevar elevados tiempos de espera y gran número de citas médicas, en la que se incluye la derivación de pacientes hacia establecimientos donde sea posible realizar una PAAF. No obstante, el recurso humano capacitado para llevar a cabo este procedimiento es escaso. ${ }^{7}$

Es de interés entrenar a médicos en la práctica de la PAAF, de manera que se pueda aumentar el acceso a este procedimiento y hacer un correcto diagnóstico en un periodo de tiempo menor. La evidencia actual ha demostrado que el entrenamiento en esta técnica se asocia a un aumento del rendimiento en la tasa diagnóstica de las biopsias tiroideas. ${ }^{8}$ El modelo educacional tradicional en este tipo de técnica consiste en la observación de un experto durante el procedimiento y la posterior práctica por parte del estudiante en un paciente real. Sin embargo, este modelo instruccional presenta dificultades técnicas y éticas relacionadas con la seguridad del paciente. ${ }^{9}$

La simulación provee de un ambiente seguro para el paciente y el estudiante, y para la adquisición de habilidades técnicas. ${ }^{10,11} \mathrm{La}$ evidencia actual sustenta que las habilidades aprendidas en simulación pueden ser transferidas a la práctica clínica. ${ }^{12-15} \mathrm{Si}$ bien existen modelos para este entrenamiento en la literatura $^{16-18}$ y en el mercado, ${ }^{19}$ éstos son aún perfectibles respecto de su costo efectividad para permitir el entrenamiento seriado de alumnos.

El objetivo de este trabajo es presentar un modelo de punción tiroidea de bajo costo y una pauta para la evaluación de este procedimiento que pueda ser aplicado tanto en el entrenamiento como en la práctica clínica.

\section{MATERIAL Y MÉTODOS}

Diseño de estudio: se realizó un estudio descriptivo de investigación en educación de habilidades técnicas basada en simulación. El presente estudio fue aprobado por el comité de ética de nuestra institución (Código Proyecto 190319013).

Desarrollo del modelo simulado: se construyó un modelo de aprendizaje simulado con material doméstico de bajo costo. El proceso de desarrollo siguió una metodología Lean ${ }^{20}$ que consiste en manufacturar un producto mínimo viable y generar mejoras consecutivas, conforme la evaluación constante por parte de potenciales usuarios finales. Se consideraron los siguientes parámetros: ecogenicidad (medido mediante ecografía con un transductor linear $10-5 \mathrm{MHz}$ SonoSite ${ }^{\circledR}$ ), consistencia, puncionabilidad, similitud anatómica, durabilidad y costos. En este proceso participaron dos endocrinólogas formadas en punción tiroidea guiada por ecografía (con experiencia en 8,000 y 200 punciones de manera aproximada, cada una) y un residente de endocrinología. Se recogió la opinión respecto de los modelos consecutivos de acuerdo con encuestas online (Apéndice 1), focus group, y entrevistas semiestructuradas breves. Se llevó registro del costo de los materiales empleados. Los materiales usados en el proceso de elaboración fueron: gelatina sin sabor (disponible en supermercados), Metamucil ${ }^{\circledR}$, fibra de psyllium en polvo, azul de metileno, bandeja de aluminio, botellas de plástico, arándanos, perlas de hidrogel, bombillas de plástico, Penrose, agua, tubos plásticos de suero fisiológico, refrigerador y balanza digital.

Desarrollo de pauta de evaluación: se construyó una pauta de evaluación para medir la calidad técnica de la punción tiroidea en el modelo simulado y en el paciente real. El proceso de selección de los aspectos técnicos y clínicos relevantes a evaluar se basó en una revisión de la literatura existente y en la opinión de expertos (dos endocrinólogas) mediante entrevistas hasta lograr un consenso. Se seleccionaron los puntos más relevantes a evaluar cuando se aplica una PAAF, considerando diferentes dominios del aprendizaje como áreas de comunicación médico-paciente y con el equipo de salud, habilidad técnica relacionada al procedimiento y verificación del estado del paciente durante y después del procedimiento. 


\section{RESULTADOS}

\section{Desarrollo del modelo simulado}

Se desarrolló un primer modelo (M1), (Figura 1) con una mezcla de gelatina (gelatina pura en polvo) y Metamucil ${ }^{\circledR}$ (disponible en casas comerciales) como base para hacer el cuello, se utilizó como molde una bandeja rectangular desechable, se seleccionaron arándanos para simular nódulos, tubo de intubación traqueal para simular tráquea y bajadas de suero para representar los vasos carotídeos. La posición de los diferentes reparos anatómicos se determinó luego de realizar mediciones en ecografías cervicales normales donde se consideró la distancia entre vasos carotídeos y tráquea como reparo anatómico principal.

El modelo M1 presentaba adecuada ecogenicidad, consistencia y puncionabilidad según los expertos, con una vida útil de dos semanas en ambiente refrigerado hasta que era invadida por hongos del ambiente. Los arándanos representaban de manera satisfactoria nódulos hiperecogénicos heterogéneos en la ecografía, pero no permitían obtener muestras y eran visibles a simple vista en el modelo. El tubo traqueal lograba una representación ecográfica correcta mientras que las bajadas de suero tenían un tamaño menor a los vasos carotídeos por lo que no eran satisfactorias.

Se desarrolló un segundo modelo (M2), (Figura 2), usando un molde semicilíndrico (media botella plástica de $500 \mathrm{~cm}^{3}$, a la que se adicionó una base en un extremo para agregar estabilidad), se cambió el Metamuci ${ }^{\circledR}$ por polvo de fibra de psyllium (por su menor contenido en azúcar) y se agregó azul de metileno a la

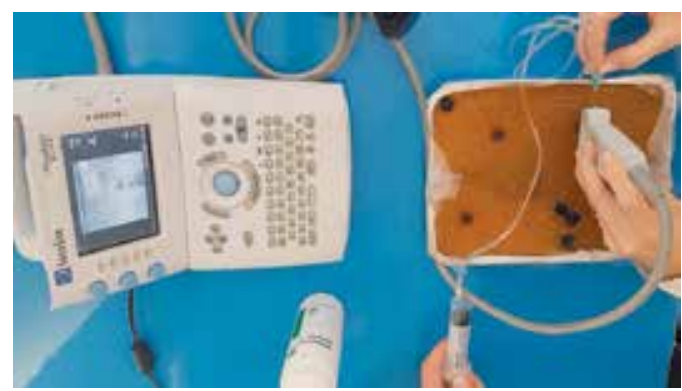

Figura 1: Modelo 1 (M1) siendo evaluado con transductor linear.
Figura 2:

Modelo 2 (M2) tiene forma semicilíndrica y los arándanos son visibles a simple vista.
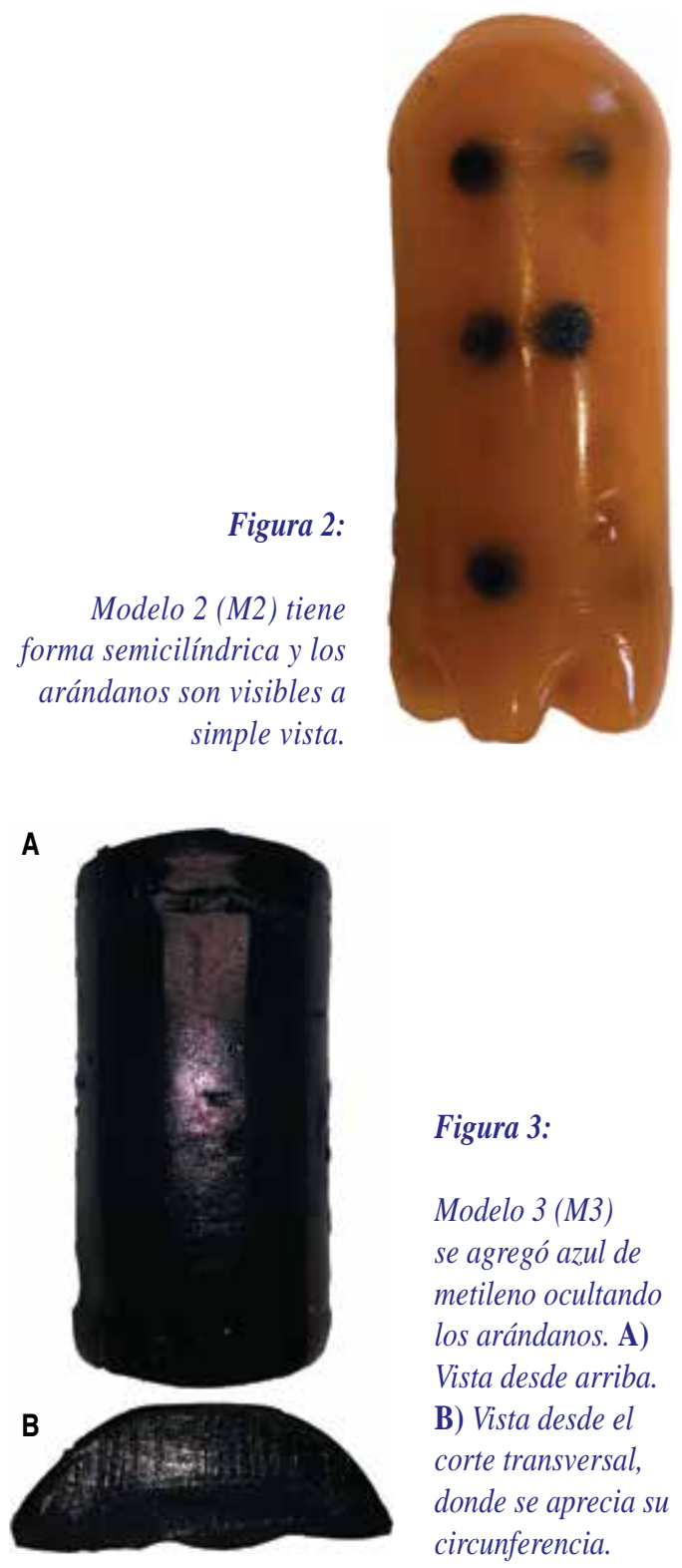

mezcla con el fin de ocultar la posición de los arándanos en el modelo. Se adicionaron perlas de hidrogel previamente hidratadas hasta un tamaño de entre 8 y $15 \mathrm{~mm}$ para emular nódulos hipoecogénicos. Se reemplazó el tubo traqueal por un tubo plástico cilíndrico de suero fisiológico vacío y sellado, para disminuir los costos, y los vasos carotídeos por drenajes Penrose ligados en ambos extremos rellenos de agua. El semicilindro logrado con $500 \mathrm{~cm}^{3}$, en opinión de expertos y residentes en entrenamiento, subestimaba la circunferencia real de un cuello. 
La sustitución por fibra de psyllium aumentó su durabilidad entre tres y cuatro semanas en condiciones refrigeradas a $4{ }^{\circ} \mathrm{C}$ y la adición de azul de metileno tuvo buena recepción por parte de los expertos, al igual que la nueva tráquea. Los drenajes, si bien lograban excelente representación ecográfica, presentaban la limitante que una vez puncionados por error, como parte del entrenamiento, se perdía el contenido y con esto la ecogenicidad, limitando la vida útil del modelo. Las perlas de hidrogel formaban una imagen ecográfica satisfactoria, pero no permiten obtener muestras en la punción y luego de alrededor de 10 punciones, se volvían hiperecogénicas. Se mantuvieron los arándanos para contar con simuladores de nódulos tiroideos de diferente ecogenicidad.

Siguiendo las sugerencias anteriores se desarrolló un tercer y final modelo (M3), (Figura 3). Se usó esta vez una botella plástica de 1,500 $\mathrm{cm}^{3}$ para aumentar la circunferencia del cuello. Se reemplazaron los drenajes por bombillas de refresco de $6 \mathrm{~mm}$ de diámetro, selladas en los extremos y rellenas de agua. El resto de los materiales se mantuvo sin cambios. Sobre este modelo los expertos estuvieron muy de acuerdo en que éste presenta similitud aceptable con un cuello humano, una ecogenicidad aceptable, una sensación al puncionar aceptable, nódulos tiroideos a la ecografía aceptables. Ambos estuvieron al menos de acuerdo en que la anatomía del cuello a la ecografía era aceptable y en total acuerdo en que el modelo era adecuado para entrenar la técnica del ecógrafo como guía y la técnica de punción por aguja fina en ecografía tiroidea. Se presentan las imágenes ecográficas

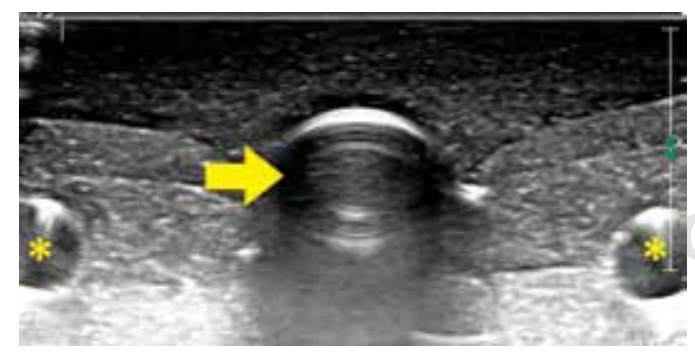

Figura 4: Imagen obtenida de M3 con transductor linear de 12-5 MHz y ecógrafo Philips Affiniti 30. La flecha indica la tráquea circundada por tejido tiroideo simulado. Los asteriscos indican los vasos carotídeos.

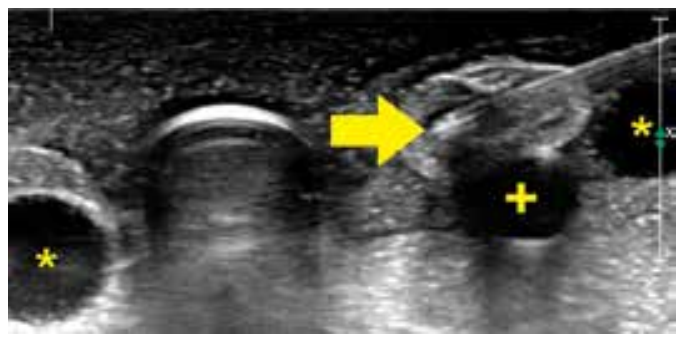

Figura 5: Imagen obtenida de M3 con transductor linear de 12-5 MHz y ecógrafo Philips Affiniti 30, durante la punción de un nódulo. La flecha indica la punta de la aguja puncionando nódulo hiperecogénico y heterogéneo (arándano). El signo “+” indica un nódulo hipoecogénico (perla de hidrogel). El asterisco indica vaso carotídeo.

del modelo en las Figuras 4 y 5 . El costo del molde y de los materiales reutilizables es de 2.089 CLP (pesos chilenos) (3,1 USD), y el costo de cada modelo descontando lo anterior es de 1.735 CLP/2,6 USD (el detalle de materiales y costos aparecen en la Tabla 1). Los pasos para la elaboración del modelo se encuentran en el Apéndice 2.

\section{Pauta de evaluación}

Se desarrolló una pauta de 12 ítems (Tabla 2). Los siete primeros corresponden a elementos técnicos y procedimentales, medidos con una escala Likert de tres puntos (bajo, medio y alto) en la que se caracteriza cada categoría. Se incluyen tres ítems clínicos que corresponden a la formación de hematoma cervical tras la punción (medido como sí/no), el dolor del paciente (escala visual análoga del 1 al 10) y suficiencia de la muestra (sí/no). Dos ítems miden el número de intentos para puncionar un mismo nódulo y si la punción múltiple fue justificada o no.

\section{DISCUSIÓN}

La simulación como herramienta educacional ha adquirido un rol fundamental en los programas de simulación médica en todo el mundo. En el mercado existen simuladores para punción tiroidea, pero su elevado costo (alrededor de 2,760 USD) limita su uso. ${ }^{19} \mathrm{En}$ 
la literatura médica se encuentran otros modelos publicados, ${ }^{16-18}$ donde se reporta el uso de éstos u otros materiales en combinación con tejidos ex vivo. Sin embargo, aún no se ha desarrollado un programa costo-efectivo para ejercitar esta competencia.

El presente modelo cuenta con diversas ventajas. En primer lugar, los materiales utilizados son de bajo costo (precio estimado por modelo: $1.735 \mathrm{CLP} / 2,6$ USD) lo que es similar a otros reportados. ${ }^{16}$ Estos materiales están disponibles de forma amplia y no se requiere de conocimientos específicos para su manipulación como podría ser el caso de productos químicos. La vida útil del modelo es de tres a cuatro semanas en recipiente hermético refrigerado; considerando el escaso gasto, es bastante costo-efectivo. Parte de sus materiales son reutilizables (molde, tráquea y vasos) y el resto son, en su mayoría, compostables, lo que es deseable debido a los altos costos y desechos que genera la simulación. ${ }^{21}$ El diseño del modelo incluye varios nódulos, por lo que puede ser utilizado por distintos alumnos en diferentes sesiones.

Nuestro modelo no está exento de limitaciones. Requiere tiempo de elaboración prolongado (alrededor de una hora), aunque éste puede ser reducido con el uso de un agitador magnético. Existe una variabilidad intermodelo dada por la dificultad para crear un modelo perfectamente anatómico, pues esta etapa es de operador dependiente. Lo anterior se refleja sobre todo al momento de posicionar los vasos carotídeos, pues éstos pueden desplazarse antes de que el modelo aumente su consistencia durante el refrigerado. Sin embargo, estas diferencias en la anatomía no entorpecen el aprendizaje según los expertos y también pueden ser evidenciadas en el paciente real.

Por otra parte, la circunferencia alcanzada con una botella de $1,500 \mathrm{~cm}^{3}(\mathrm{M} 3)$, pese a mejorar con respecto a $\mathrm{M1}$, aún refleja un cuello delgado, situación cada vez más infrecuente con el aumento de la obesidad en la población. La punción de los nódulos deja trayectos hiperecogénicos en la mezcla, pero éstos tienden a ser borrados si el modelo es calentado en un microondas a media-baja potencia por 10 o 30 segundos y luego refrigerado de nuevo. El hecho de que nuestro modelo no cuente con lóbulos tiroideos delimitados hace que el número de punciones esté dado por la cantidad de nódulos añadidos durante su elaboración. Los nódulos hechos de arándanos no presentan límite de punciones y los de hidrogel alrededor de 10 punciones, dado que pierden su ecogenicidad.

La pauta de evaluación es un instrumento fundamental a la hora de valorar la efectividad del entrenamiento en el modelo simulado. ${ }^{22}$ La pauta desarrollada se puede dividir en dos secciones, una parte que permite evaluar el entrenamiento en el modelo y otra, para medir la

\begin{tabular}{lcc} 
& Tabla 1: Materiales y costos. & \\
& Precio total/por modelo (CLP) & Precio en USD \\
\hline Costo de materiales reutilizables & & \\
Botella plástica $1,500 \mathrm{~cm}^{3}$ & 1.650 & 2,4 \\
Frasco cilíndrico suero & 400 & 0,6 \\
Bombillas (20 un.) & $390 / 39(2$ un.) & $0,6 / 0,06$ \\
Total & 2.089 & 3,1 \\
Costos por modelo & & $12,5 / 0,5$ \\
Polvo de fibra de psyllium (200 g) & $8.470 / 300(7 \mathrm{~g})$ & $1 / 1,5$ \\
Gelatina 4 sobres (7.5 g) & $650 / 975(6 \mathrm{sobres})$ & $2,6 / 0,3$ \\
Azul de metileno $\%$ (20 mL) & $1.760 / 176(2 \mathrm{~mL})$ & $2,5 / 0,3$ \\
Arándanos (125 g-82 un. Aprox.) & $1.690 / 164(8 \mathrm{un})$. & $2,2 / 0,2$ \\
Perlas de hidrogel (100 un.) & $1.500 / 120(8 \mathrm{un})$. & 2,6 \\
Total & 1.735 & \\
\hline
\end{tabular}

un. $=$ Unidad 
Tabla 2: Pauta de evaluación.

Ítem

Descripción de la categoría

\section{Elementos a evaluar durante el entrenamiento}

1. Preparación del campo

2. Uso del ecógrafo

3. Emplanamiento del agua y ecógrafo

4. Tiempo y número de punciones

5. Toma de muestra (en el caso de usar modelo que permita obtener muestra)

6. Número de punciones (intentos en un mismo nódulo)

\section{Elementos a evaluar en la práctica con paciente real}

7. Comunicación con el paciente

8. Instrucción al ayudante

9. Dolor del paciente

10. Hematoma

11. Muestra suficiente

12. Punción múltiple justificada estructuras vasculares cinco intentos excursión con aguja

Valorar sí/no
Bajo: preparación inadecuada del campo, técnica estéril deficiente, se contamina Medio: preparación adecuada del campo, tarda más del tiempo esperado Alto: destreza en preparación del campo y mantiene técnica estéril Bajo: no logra ubicar nódulos, no ocupa Doppler para ubicar estructuras vasculares Medio: localiza nódulos, es incapaz de fijar imagen sobre éstos, no ubica

Alto: reconoce de forma adecuada reparos anatómicos y localiza y fija nódulos. Reconoce de forma correcta estructuras vasculares

Bajo: no logra ver aguja ni punta de aguja en plano de ecografía junto al nódulo Medio: emplanar aguja y nódulo de manera inconsistente Alto: logra emplanar aguja y nódulo de forma consistente Bajo: tarda más de lo esperado, hace pausas constantemente, realiza más de

Medio: poco eficiente, requiere más de un intento

Alto: mínimo tiempo, eficiente y obtiene muestra al primer intento Bajo: no logra obtener muestra, no realiza movimientos al interior del nódulo Medio: obtiene muestra escasa o en múltiples punciones y realiza incursión y

Alto: realiza incursión y excursión hasta obtener suficiente muestra en una punción Valorar en escala continua. Ejemplo 1, 2, 3... 10

Bajo: no explica procedimiento ni complicaciones

Medio: explica de manera parcial procedimiento y complicaciones

Alto: explica procedimiento, complicaciones y obtiene consentimiento informado

Bajo: no da instrucciones a ayudante

Medio: omite registro de imágenes o aspiración

Alto: solicita registro correcto de imagen, aspirar y liberar aspiración

Valorar en escala visual análoga de 1 a 10

Valorar formación de hematoma postpunción en el paciente (sí/no)

Valoración histopatológica de la muestra. Suficiente sí/no calidad del procedimiento en un paciente real. Esta característica de la pauta es una ventaja, pues podría ser aplicada en la simulación y en el ambiente clínico.

\section{CONCLUSIÓN}

En este trabajo se presenta un modelo de punción tiroidea de bajo costo y de fácil replicabilidad junto a una pauta para evaluar el entrenamiento. La proyección a futuro de este modelo está vinculada al desarrollo de un programa de entrenamiento validado para punción tiroidea guiada con ecografía, el cual será evaluado mediante la pauta desarrollada que permitirá medir, tanto el aprendizaje en el modelo como su potencial transferencia de habilidades al paciente real. 


\section{REFERENCIAS}

1. Dean DS, Gharib H. Epidemiology of thyroid nodules. Best Pract Res Clin Endocrinol Metab. 2008; 22 (6): 901-911. doi: 10.1016/J.BEEM.2008.09.019.

2. Desforges JF, Mazzaferri EL. Management of a solitary thyroid nodule. N Engl J Med. 1993; 328 (8): 553-559. doi: 10.1056/NEJM199302253280807.

3. Guth S, Theune U, Aberle J, Galach A, Bamberger CM. Very high prevalence of thyroid nodules detected by high frequency $\left(13^{\circ} \mathrm{MHz}\right)$ ultrasound examination. Eur J Clin Invest. 2009; 39 (8): 699-706. doi: 10.1111/j.1365-2362.2009.02162.x.

4. Mazzaferri EL. Thyroid cancer in thyroid nodules: Finding a needle in the haystack. Am J Med. 1992; 93 (4): 359-362. doi: 10.1016/0002-9343(92)90163-6.

5. Burman KD, Wartofsky L. Thyroid Nodules. N Engl J Med. 2016; 374 (13): 1294-1295. doi: 10.1056/ NEJMc1600493.

6. Haugen BR, Alexander EK, Bible KC, et al. 2015 American Thyroid Association Management Guidelines for Adult Patients with Thyroid Nodules and Differentiated Thyroid Cancer: The American Thyroid Association Guidelines Task Force on Thyroid Nodules and Differentiated Thyroid Cancer. Thyroid. 2016; 26 (1): 1-133. doi: 10.1089/thy.2015.0020.

7. Fernández-García JC, Mancha-Doblas I, OrtegaJiménez MV, et al. Estructura diagnóstica y funcional de una consulta de alta resolución de nódulo tiroideo. Endocrinol y Nutr. 2014; 61 (6): 329-334. doi: 10.1016/j.endonu.2013.09.004.

8. Beland MD, Anderson TJT, Atalay MK, Grand DJ, Cronan JJ. Resident experience increases diagnostic rate of thyroid fine-needle aspiration biopsies. Acad Radiol. 2014; 21 (11): 1490-1494. doi: 10.1016/j. acra.2014.06.006

9. Reznick RK, MacRae H. Teaching surgical skills-changes in the wind. N Engl J Med. 2006; 355 (25): 2664-2669. doi: 10.1056/NEJMra054785.

10. Aggarwal R, Mytton OT, Derbrew M, et al. Training and simulation for patient safety. Qual Saf Heal Care. 2010; 19 (Suppl 2): i34-i43. doi: 10.1136/ qshc.2009.038562.

11. Choy I, Okrainec A. Simulation in surgery: perfecting the practice. Surg Clin North Am. 2010; 90 (3): 457473. doi: 10.1016/j.suc.2010.02.011.

12. Boza C, León F, Buckel E, et al. Simulation-trained junior residents perform better than general surgeons on advanced laparoscopic cases. Surg Endosc. 2017; 31 (1): 135-141. doi: 10.1007/s00464-016-4942-6.

13. Al-Kadi AS, Donnon T, Oddone-Paolucci E, Mitchell $P$, Debru E, Church N. The effect of simulation in improving students' performance in laparoscopic surgery: a meta-analysis. Surg Endosc. 2012; 26 (11): 3215-3224. doi: 10.1007/s00464-012-2327-z.

14. Buckley CE, Kavanagh DO, Traynor O, Neary PC. Is the skillset obtained in surgical simulation transferable to the operating theatre? Am J Surg. 2014; 207 (1): 146-157. doi: 10.1016/j.amjsurg.2013.06.017.

15. Dawe SR, Windsor JA, Broeders JA, Cregan PC, Hewett PJ, Maddern GJ. A systematic review of surgical skills transfer after simulation-based training: laparoscopic cholecystectomy and endoscopy. Ann Surg. 2014; 259 (2): 236-248.

16. Baba M, Matsumoto K, Yamasaki N, et al. Development of a tailored thyroid gland phantom for fine-needle aspiration cytology by three-dimensional printing. J Surg Educ. 2017; 74 (6): 1039-1046. doi: 10.1016/j. jsurg.2017.05.012.

17. Urbina S, Balcells A, Avaria P, Hirsch M. Fantoma para punción tiroidea ecoguiada, de elaboración casera y bajo costo. Rev Argentina Radiol. 2017; 81 (2): 122128. doi: 10.1016/J.RARD.2017.05.002.

18. Richardson C, Bernard S, Dinh VA. A cost-effective, gelatin-based phantom model for learning ultrasoundguided fine-needle aspiration procedures of the head and neck. J Ultrasound Med. 2015; 34 (8): 1479-1484. doi: 10.7863/ultra.34.8.1479.

19. Thyroid biopsy ultrasound training phantom model. https://www.bluephantom.com/product/ThyroidBiopsy-Ultrasound-Training-Model.aspx? cid $=413$. Accessed June 27, 2019.

20. Clark DM, Silvester K, Knowles S. Lean management systems: creating a culture of continuous quality improvement. J Clin Pathol. 2013; 66 (8): 638-643. doi: 10.1136/jclinpath-2013-201553.

21. Zendejas B, Wang AT, Brydges R, Hamstra SJ, Cook DA. Cost: the missing outcome in simulation-based medical education research: a systematic review. Surgery. 2013; 153 (2): 160-176. doi: 10.1016/j.surg.2012.06.025.

22. Cook DA, Beckman TJ. Current concepts in validity and reliability for psychometric instruments: theory and application. Am J Med. 2006; 119 (2): 166.e7-166. e16. doi: 10.1016/j.amjmed.2005.10.036.

Correspondencia:

Dra. Nicole Marie Lustig Franco

Departamento de Endocrinología, Facultad de Medicina,

Pontificia Universidad Católica de Chile.

Diagonal Paraguay Núm. 362, cuarto piso, Santiago, Chile.

Fono/fax: +5692-2354-3095

E-mail: nmlustig@uc.cl 